$\mathrm{e}^{+} \mathrm{e}^{-}$Collisions from Phi to Psi 2013 (PHIPSI2013)

International Journal of Modern Physics: Conference Series

Vol. 35 (2014) 1460428 (5 pages)

(C) The Author

DOI: $10.1142 /$ S2010194514604281

\title{
CHARMONIUM(-LIKE) SPECTROSCOPY AND DECAYS FROM BESIII
}

\author{
LIANG YAN \\ for the BESIII collaboration \\ Department of Modern Physics, University of Science and Technology of China \\ HeFei, AnHui, China \\ yanl@ihep.ac.cn
}

Published 18 December 2014

\begin{abstract}
The recent results about charmonium and charmonium-like spectroscopy and decays from BESIII are presented in this talk. We report the observation of $X(3872)$ from $Y(4260)$ radiative transition, $\pi^{+} \pi^{-} h_{c}$ line shape measurement and $Z_{c}$ states. In addition, the accurate measurement of $\eta_{c}$ parameters, the analysis on $h_{c}$ exclusive decays into $\gamma \eta_{c}$, and the first observation of the M1 transition $\psi^{\prime} \rightarrow \gamma \eta_{c}(2 S)$ are included in this talk.
\end{abstract}

Keywords: Charmonium; XYZ; BESIII.

PACS Numbers: 14.40.Pq, 13.25.Gv, 13.40.Hq

\section{Introduction}

In 2009, the upgraded Beijing spectrometer (BESIII) ${ }^{1}$ at Beijing Electron Positron Collider (BEPCII) started to take data. Recently, a lot of data at different energy points above the $4 \mathrm{GeV}$ are taken for studying the charmoniumlike states, such as $X(3872), Z_{c}$ states. The results about $\eta_{c}, h_{c}$ and $\eta_{c}(2 S)$ in this talk are based on the $1.06 \times 10^{8} \psi^{\prime}$ event data sample.

\section{Charmonium states}

The $\eta_{c}$ is the lowest-lying state of the $c \bar{c}$ system, but its properties are not well measured. The $\eta_{c}$ is reconstructed through six different channels: $\psi^{\prime} \rightarrow$ $\gamma \eta_{c}, \eta_{c} \rightarrow X_{i}, X_{i}=\left(K_{S}^{0} K^{ \pm} \pi^{\mp}, K^{+} K^{-} \pi^{0}, \pi^{+} \pi^{-} \eta, K_{S}^{0} K^{ \pm} \pi^{\mp} \pi^{+} \pi^{-}, 3\left(\pi^{+} \pi^{-}\right)\right.$, $\left.K^{+} K^{-} \pi^{+} \pi^{-} \pi^{0}\right){ }^{2}$ as shown in Fig. 1. A simultaneous fit that incorporates interference between the signal reaction and a non-resonant radiative background determine the mass and width of $\eta_{c}$ to be $m_{\eta_{c}}=(2984.3 \pm 0.6 \pm 0.6) \mathrm{MeV} / c^{2}$ and

This is an Open Access article published by World Scientific Publishing Company. It is distributed under the terms of the Creative Commons Attribution 3.0 (CC-BY) License. Further distribution of this work is permitted, provided the original work is properly cited. 

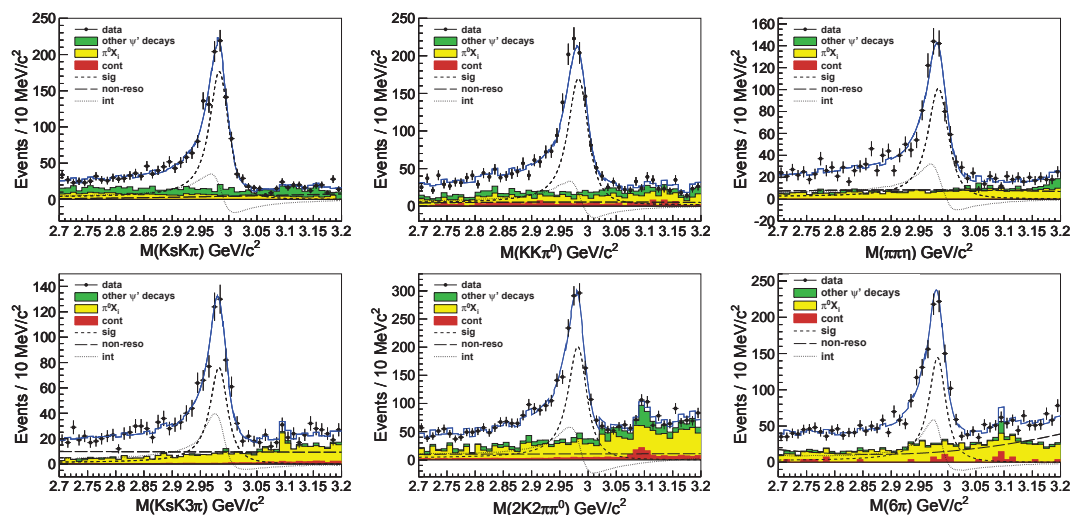

Fig. 1. The invariant mass distributions in $\psi^{\prime} \rightarrow \gamma \eta_{c}$ for the decays $K_{S}^{0} K^{ \pm} \pi^{\mp}, K^{+} K^{-} \pi^{0}$, $\pi^{+} \pi^{-} \eta, K_{S}^{0} K^{ \pm} \pi^{\mp} \pi^{+} \pi^{-}, K^{+} K^{-} \pi^{+} \pi^{-} \pi^{0}$, and $3\left(\pi^{+} \pi^{-}\right)$, respectively, with the fit results (constructive) superimposed.

$\Gamma_{\eta_{c}}=(32.0 \pm 1.2 \pm 1.0) \mathrm{MeV}$. The new BESIII results are so far the most precise measurement of the properties of $\eta_{c}$, which agree with the values found in photonphoton fusion and $B$ decays.

The $h_{c}$ is the $\mathrm{P}$-wave spin singlet charmonium below open-charm threshold. It could be studied through the mode of $\psi^{\prime} \rightarrow \pi^{0} h_{c}, h_{c} \rightarrow \gamma \eta_{c}$. In BESIII, sixteen kinds of exclusive hadronic final states are used to reconstruct $\eta_{c}{ }^{3}$ To extract the $h_{c}$ resonant parameters and the yield for each $\eta_{c}$ decay channel, the sixteen $\pi^{0}$ recoil mass distributions are fitted simultaneously with a binned maximum likelihood method.

$\eta_{c}(2 S)$, the first radial excitation of $\eta_{c}$, is poorly understood relative to the other charmonium below open-charm threshold. In BESIII, $\eta_{c}(2 S)$ was observed with the transition of $\psi^{\prime} \rightarrow \gamma \eta_{c}(2 S), \eta_{c}(2 S) \rightarrow K_{S}^{0} K^{ \pm} \pi^{\mp}$ and $K^{+} K^{-} \pi^{0} \cdot{ }^{4}$ Recently, the evidence of $\eta_{c}(2 S) \rightarrow K_{S}^{0} K^{ \pm} \pi^{\mp} \pi^{+} \pi^{-5}$ is reported with the product branching fraction $\mathcal{B}\left(\psi^{\prime} \rightarrow \gamma \eta_{c}(2 S), \eta_{c}(2 S) \rightarrow K_{S}^{0} K^{ \pm} \pi^{\mp} \pi^{+} \pi^{-}\right)=(7.03 \pm 2.10 \pm 0.70) \times 10^{-6}$.

\section{Charmonium-like states}

The $X(3872)$ was observed by Belle in $B^{ \pm} \rightarrow K^{ \pm} \pi^{+} \pi^{-} J / \psi$ decays. ${ }^{6}$ In BESIII, we report the observation of $X(3872)$ in the channel of $e^{+} e^{-} \rightarrow \gamma X(3872),{ }^{7} X(3872) \rightarrow$ $\pi^{+} \pi^{-} J / \psi$ based on the accumulated data taken at $4.009,4.230,4.260,4.360 \mathrm{GeV}$. Fig. 2 shows the fit of the invariant mass of $\pi^{+} \pi^{-} J / \psi$. The measured mass of $X(3872)$ is $(3872.1 \pm 0.8 \pm 0.2) \mathrm{MeV} / c^{2}$, and the statistical significance is $5.2 \sigma$.

In BESIII, we study the $e^{+} e^{-} \rightarrow \pi^{+} \pi^{-} h_{c}$ at thirteen center of mass energy points from 3.900 to $4.420 \mathrm{GeV}^{8}$ In order to include more $h_{c}$ signals, sixteen exclusive hadronic final states are used: $p \bar{p}, \pi^{+} \pi^{-} \pi^{+} \pi^{-}, K^{+} K^{-} \pi^{+} \pi^{-}$, $p \bar{p} \pi^{+} \pi^{-}, K^{+} K^{-} \pi^{+} \pi^{-} \pi^{+} \pi^{-}, \pi^{+} \pi^{-} \pi^{+} \pi^{-} \pi^{+} \pi^{-}, K^{+} K^{-} \pi^{+} \pi^{-} \pi^{+} \pi^{-}, K_{S}^{0} K^{ \pm} \pi^{\mp}$, $K_{S}^{0} K^{ \pm} \pi^{\mp} \pi^{+} \pi^{-}, K^{+} K^{-} \pi^{0}, p^{+} p^{-} \pi^{0}, \pi^{+} \pi^{-} \eta, K^{+} K^{-} \eta, \pi^{+} \pi^{-} \pi^{+} \pi^{-} \eta, \pi^{+} \pi^{-} \pi^{0} \pi^{0}$, 


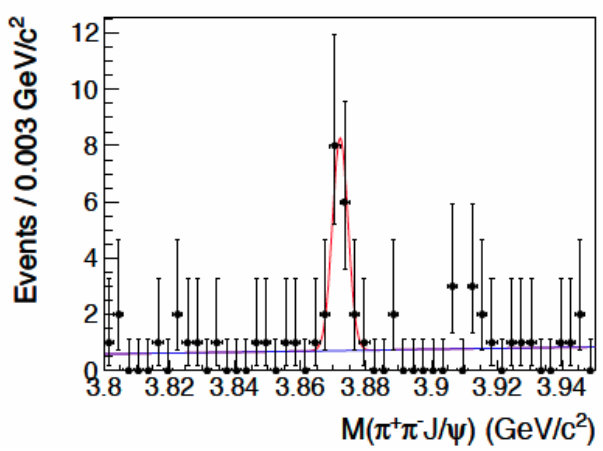

Fig. 2. Fit to the invariant mass of $\pi^{+} \pi^{-} J / \psi$ with MC simulated histogram convulsing a Gaussian function for signal and a linear background term. Dots with error bars are data, the curves are the best fit.

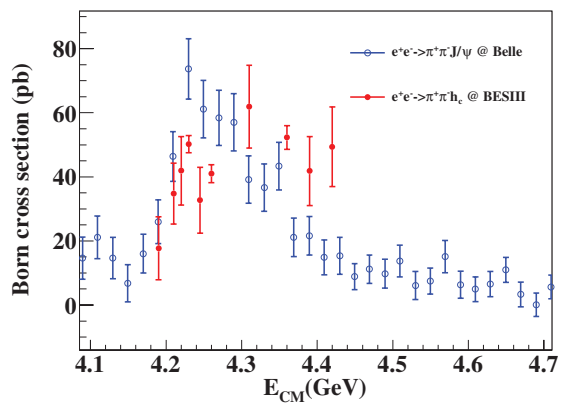

Fig. 3. The cross section of $\pi^{+} \pi^{-} h_{c}$. Red dots with error bars for BESIII and blue circles with error bars for BELLE.

and $\pi^{+} \pi^{-} \pi^{+} \pi^{-} \pi^{0} \pi^{0}$. The cross sections are shown in Fig 3, and the line shape is different from the Belle measurement on $e^{+} e^{-} \rightarrow \pi^{+} \pi^{-} J / \psi$.

We observe a structure around $3.9 \mathrm{GeV} / c^{2}$ in the $\pi^{ \pm} J / \psi$ mass spectrum from the process of $e^{+} e^{-} \rightarrow \pi^{+} \pi^{-} J / \psi \cdot{ }^{9}$ As the Fig. 4 shows, a fit yields a mass of $(3899.0 \pm 3.6 \pm 4.9) \mathrm{MeV} / c^{2}$, a width of $(46 \pm 10 \pm 20) \mathrm{MeV}$, and larger than $8 \sigma$ statistical significance. Its production ratio is measured to be $R=$ $\frac{\sigma\left(e^{+} e^{-} \rightarrow \pi^{ \pm} Z_{c}(3900)^{\mp} \rightarrow \pi^{+} \pi^{-} J / \psi\right)}{\sigma\left(e^{+} e^{-} \rightarrow \pi^{+} \pi^{-} J / \psi\right)}=(21.5 \pm 3.3 \pm 7.5) \%$. This state denoted $Z_{c}(3900)$, has a strong coupling to charmonium and electronic charge, so it cannot be interpreted as a conventional $c \bar{c}$ state.

By analyzing the Dalitz plot of $\pi^{+} \pi^{-} h_{c}$ system in the $e^{+} e^{-} \rightarrow \pi^{+} \pi^{-} h_{c}$ process, ${ }^{8}$ we observe a narrow structure close to the $\left(D^{*} \bar{D}^{*}\right)^{ \pm}$threshold in the $\pi^{ \pm} h_{c}$ mass spectrum. As the Fig. 5 shows, fitting to the $\pi^{ \pm} h_{c}$ mass spectrum at $4.230,4.260$ and $4.360 \mathrm{GeV}$ simultaneously, yields a mass of $(4022.9 \pm 0.8 \pm 2.7) \mathrm{MeV} / c^{2}$ and a width of $(7.9 \pm 2.7 \pm 2.6) \mathrm{MeV}$. Similar to the $Z_{c}(3900)$ observed in the $\pi^{ \pm} J / \psi$ mass 


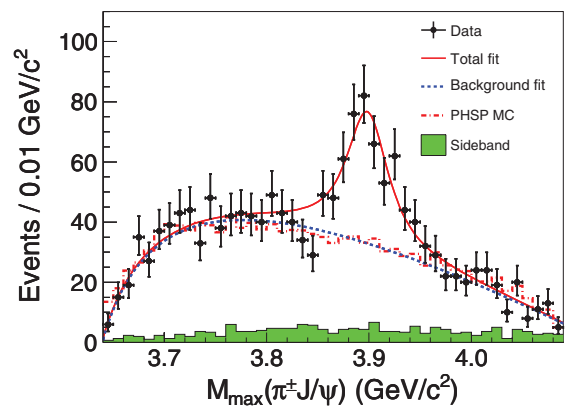

Fig. 4. Unbinned maximum likelihood fit to the distribution of the $M_{\max }(\pi J / \psi)$. Points with error bars are data, the curve are the best fit, the dashed histogram are the phase space distribution and the shaded histograms is the non- $\pi^{+} \pi^{-} J / \psi$ background estimated from the normalized $J / \psi$ sideband.

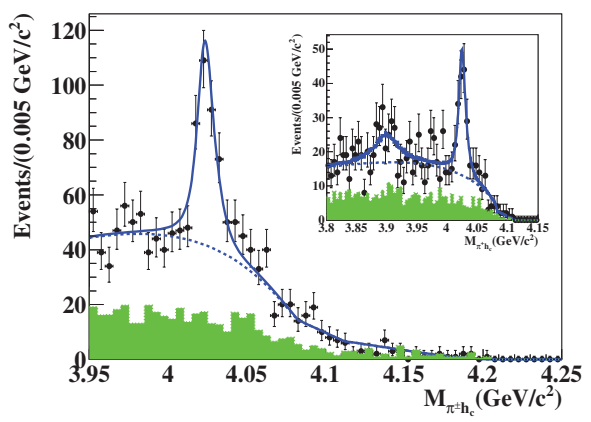

Fig. 5. Sum of the simultaneous fits to the invariant mass spectrum of $\pi^{ \pm} h_{c}$ at $4.230 \mathrm{GeV}$, $4.260 \mathrm{GeV}$ and $4.360 \mathrm{GeV}$; the inset shows the sum of the simultaneous fit to the $\pi^{+} h_{c}$ mass distribution at $4.230 \mathrm{GeV}$ and $4.260 \mathrm{GeV}$ with $Z_{c}(3900)$ and $Z_{c}(4025)$. Dots with error bars are data; shaded histograms are normalized sideband background; the solid curves show the total fit, and the dotted curves the background from the fit.

spectrum, this structure also couples to charmonium and has an electric charge, which is suggestive a state containing more quarks than just a pair of charm quark. There is no significant signal for $Z_{c}(3900) \rightarrow \pi^{ \pm} h_{c}$ and the production cross section is found to be smaller than $11 \mathrm{pb}$ at $90 \%$ confidence level at $4.260 \mathrm{GeV}$, which is lower than that of $Z_{c}(3900) \rightarrow \pi^{ \pm} J / \psi$.

Using a partial reconstruction technique, BESIII also studies the process $e^{+} e^{-} \rightarrow$ $\left(D^{*} \bar{D}^{*}\right)^{ \pm} \pi^{\mp}$ at $4.260 \mathrm{GeV}$ using $827 \mathrm{pb}^{-1}$ data sample. ${ }^{10}$ In Fig. 6, a structure near the $\left(D^{*} \bar{D}^{*}\right)^{ \pm}$threshold in the $\pi^{\mp}$ recoil mass spectrum is observed, which is denoted as the $Z_{c}(4025)$. The mass and width are measured to be $(4026.3 \pm 2.6 \pm 3.7) \mathrm{MeV} / c^{2}$ and $(24.8 \pm 5.6 \pm 7.7) \mathrm{MeV}$, respectively. The Born cross section is measured to be $(139 \pm 9 \pm 15) \mathrm{pb}$, and the production ratio $\frac{\sigma\left(e^{+} e^{-} \rightarrow Z_{c}(4025)^{ \pm} \pi^{\mp} \rightarrow\left(D^{*} \bar{D}^{*}\right)^{ \pm} \pi^{\mp}\right)}{\sigma\left(e^{+} e^{-} \rightarrow\left(D^{*} \bar{D}^{*}\right)^{ \pm} \pi^{\mp}\right)}$ is determined to be $0.65 \pm 0.09 \pm 0.06$. The parameters of $Z_{c}(4020)$ agree with those 


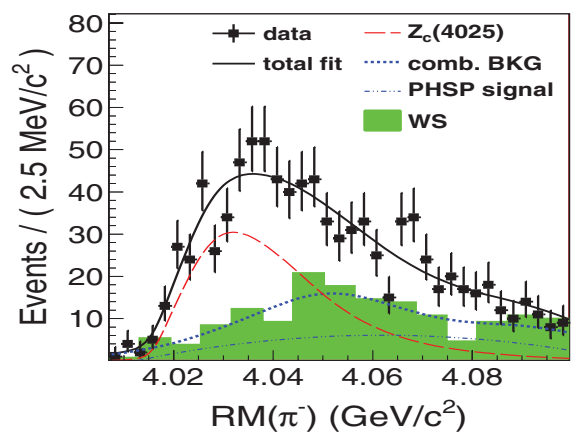

Fig. 6. Unbinned maximum likelihood fit to the $\pi^{-}$recoil mass spectrum in data.

of $Z_{c}(4025)$ within $1.5 \sigma$. Further study will help us to understand whether they are the same sate.

\section{Summary}

In summary, the charmonium states are studied based on the largest $\psi^{\prime}$ data sample, including $\eta_{c}$ parameter measurement with high precision, the analysis on $h_{c}$ exclusive decays into $\gamma \eta_{c}$, and the evidence of $\eta_{c}(2 S)$ decay to $K_{S}^{0} K^{ \pm} \pi^{\mp} \pi^{+} \pi^{-}$. For studying the XYZ states, the $X(3872)$ has been observed through $e^{+} e^{-} \rightarrow \gamma \pi^{+} \pi^{-} J / \psi$, and the line shape of $\pi^{+} \pi^{-} h_{c}$ has been measured in BESIII. The charged charmoniumlike stats, $Z_{c}(3900), Z_{c}(4020)$, and $Z_{c}(4025)$ has been observed, which may be new type of hadrons.

\section{Acknowledgments}

We would like to thank the organizers for the successful conference. And thank all the colleagues in BESIII contributing on these nice work.

\section{References}

1. BESIII Collab.(M. Ablikim et al.), Nucl. Instrum. Methods Phys. Res. Section A 614, 345 (2010).

2. BESIII Collab.(M. Ablikim et al.), Phys. Rev. Lett. 108, 222002 (2012).

3. BESIII Collab.(M. Ablikim et al.), Phys. Rev. D. 86, 092009 (2012).

4. BESIII Collab.(M. Ablikim et al.), Phys. Rev. Lett. 109, 042003 (2012)

5. BESIII Collab.(M. Ablikim et al.), Phys. Rev. D. 87, 052005 (2013).

6. Belle Collab. (S. K. Choi et al.) Phys. Rev. Lett. 91, 262001 (2003).

7. BESIII Collab.(M. Ablikim et al.) arXiv: 1310. 4101.

8. BESIII Collab.(M. Ablikim et al.) Phys. Rev. Lett. (in press).

9. BESIII Collab.(M. Ablikim et al.), Phys. Rev. Lett. 110, 252001 (2013).

10. BESIII Collab.(M. Ablikim et al.) arXiv: 1308. 2760. 